\title{
TV/Series
}

15 | 2019

La Sérialité en question(s)

\section{Les épisodes autonomes : écarts formels et narratifs dans The X-Files et Buffy The Vampire Slayer}

\section{Elaine Després}

\section{(2) OpenEdition}

Journals

Édition électronique

URL : http://journals.openedition.org/tvseries/3420

DOI : $10.4000 /$ tvseries.3420

ISSN : 2266-0909

Éditeur

GRIC - Groupe de recherche Identités et Cultures

Référence électronique

Elaine Després, «Les épisodes autonomes : écarts formels et narratifs dans The X-Files et Buffy The Vampire Slayer », TV/Series [En ligne], 15 | 2019, mis en ligne le 16 juillet 2019, consulté le 20 juillet 2019. URL : http://journals.openedition.org/tvseries/3420 ; DOI : 10.4000/tvseries.3420

Ce document a été généré automatiquement le 20 juillet 2019.

\section{(c) (i) (3)}

TV/Series est mis à disposition selon les termes de la licence Creative Commons Attribution - Pas d'Utilisation Commerciale - Pas de Modification 4.0 International. 


\title{
Les épisodes autonomes : écarts formels et narratifs dans The X-Files et Buffy The Vampire Slayer
}

\author{
Elaine Després
}

1 Les genres jouent un rôle important dans la conception, la réception et l'analyse des séries télévisées. Ils imposent aux créateurs des contraintes et permettent de constituer un auditoire fidèle (c'est le plaisir générique), compétent (par une bonne compréhension des codes), exigeant (avec un horizon d'attente clair). Ils permettent aussi aux critiques d'étudier ces œuvres immenses (parfois des centaines d'heures de télévision pour une seule série) par des approches sémiotiques, structurelles, narratologiques, de réception, etc. La question du genre en série télévisée est particulièrement complexe, puisqu'elle s'inscrit à la fois dans des considérations intrinsèques liées à l'histoire et au format du média lui-même (sitcom, telenovela, minisérie, etc.), mais aussi à des critères extrinsèques, qui la replacent dans une histoire plus large des genres littéraires et cinématographiques (mélodrame, comédie, tragédie, mais aussi science-fiction, policier, etc.).

2 À propos du genre plus strictement télévisuel, Aubry propose une nomenclature des séries, qu'elle classe entre les séries à épisodes fermés, à épisodes ouverts et les séries mixtes, prenant en compte les spécificités locales et historiques ${ }^{1}$. De son côté, Jean-Pierre Esquenazi parle de séries immobiles et de séries évolutives, mais la typologie utilisée par Stéphane Benassi et empruntée à Noël Nel semble plus opératoire, permettant de penser l'évolution des genres. Benassi utilise plus ou moins les mêmes critères formels qu'Aubry ou Esquenazi et propose de nommer «feuilleton canonique», la "forme fictionnelle narrative dont l'unité diégétique est fragmentée en plusieurs épisodes d'égale longueur ${ }^{2}$ ", et « série canonique » la « forme fictionnelle narrative dont chaque épisode possède sa propre unité diégétique et dont le(s) héros ou les thèmes sont récurrents d'un épisode à l'autre $^{3}$ ». En découlent deux cas hybrides: les «séries feuilletonnantes» (qui ont une plus grande part de sérialité) et les « feuilletons sérialisants » (avec une part plus grande 
de feuilleton $)^{4}$. En retour, cette hybridité peut mener à l'émergence de ce que je propose de nommer les «épisodes autonomes».

De l'épisode proposant une multiplicité de points de vue - à l'image du film Rashomon (Akira Kurosawa, 1950) -, aux boucles temporelles à la Groundhog Day (Harold Ramis, 1993), du pastiche aux mondes parallèles, les séries qui hybrident le feuilleton et la série proposent chaque saison plusieurs épisodes autonomes, au récit clos, qui se démarquent par leur caractère métafictionnel, expérimental et souvent ludique. Ces épisodes, par leur décalage formel et narratif par rapport à matrice de la série, sa structure et son style habituel, permettent aux personnages de prendre un détour au cours duquel un manque ou une rupture spectaculaires (manque de son, de vie, de continuité, de temps; rupture avec la formule, les conventions) les sort de l'histoire, de leur parcours prévisible.

Pour autant, les épisodes autonomes sont-ils réellement indépendants de la série dont ils sont issus ? En effet, ils font partie, malgré les apparences, du feuilleton, du macro-récit, en ce qu'ils révèlent beaucoup d'informations sur l'intrigue, la psychologie des personnages, les structures sociales, etc.; des informations impossibles à révéler dans le contexte habituel de la série, dont la grande majorité des épisodes doivent respecter la matrice. Ainsi, ces épisodes qui sortent de la matrice (à la fois celle du feuilleton et de la série) peuvent trouver une certaine liberté dans l'écart.

5 Afin d'observer ce phénomène formel, deux séries hybrides apparaissent exemplaires: The X-Files (Fox, 1993-2002) et Buffy the Vampire Slayer (The WB > UPN, 1997-2003). C'est donc à partir de celles-ci que je propose dans cet article d'explorer la notion d'épisode autonome, son rapport aux genres télévisuels et leurs architextes, son apparition et son importance historique dans les séries hybrides, mais surtout les différentes formes et thèmes récurrents qu'il emprunte au point de parfois devenir une véritable figure imposée. Pour ce faire, je commencerai par rappeler les fondements théoriques de la narration complexe des séries des trois dernières décennies, avant me focaliser sur le contexte de production et d'écriture de The X-Files et de Buffy, pour enfin catégoriser leurs épisodes autonomes et souligner leurs particularités.

\section{Les genres télésériels, leurs formules et leurs matrices}

Si la question du genre et de l'itération est fondamentale, l'étude de la formule et de la matrice qui les sous-tendent est incontournable. Selon Esquenazi :

Une formule [est] [...] un ensemble de spécifications narratives qui déterminent un usage caractéristique d'un genre. Chaque formule représente une matrice capable d'engendrer des incarnations analogues d'un modèle générique particulier [...]. Sa formule caractérise une série, mais ne la définit pas entièrement. [...] Elle représente cependant une charpente très suggestive [...] [et permet] de comprendre comment les séries déploient les structures narratives génériques d'une façon souvent originale. Les formules ont deux aspects: d'une part, une formule est attachée à un genre; d'autre part, [elle] développe et fixe des éléments qui lui sont particuliers ${ }^{5}$.

Or, cette « formule » sérielle qu'identifie Esquenazi est un peu réductrice. Elle permet une lecture générique d'une série, mais échoue à en explorer les spécificités, la richesse, les raisons de sa sérialité. La notion de "matrice " est ainsi beaucoup plus utile pour comprendre les épisodes autonomes. Selon Stéphane Benassi :

Chaque occurrence d'une série ou chaque épisode d'un feuilleton peut être considéré comme un objet unique et différent de tous ceux qui constituent l'« 
ensemble » dont il est issu, produit à partir d'une matrice (on pourrait aussi dire une trame ou un motif) initiale dont il est non pas une copie parfaite, mais une variation (dans le cas de la mise en série) ou une suite (dans le cas du feuilleton) ${ }^{6}$.

(1)

Or, la transformation d'une matrice en épisodes de feuilleton ou de série joue sur un ensemble de variations et d'invariants, opposés pour les deux genres. Pour Nel, la mise en feuilleton est «une opération de dilatation et de complexification de la diégèse, un étirement syntagmatique du récit qui conserve l'écoulement inéluctable du temps'.» Benassi ajoute que cette mise en feuilleton permet la variation sémantique, temporelle et narrative, alors même qu'elle impose une stabilité spatiale et discursive. Du côté de la mise en série, ce sont les schémas narratifs, certains paramètres sémantiques (axiologiques et idéologiques) et temporels qui demeurent invariables, alors que les lieux, les milieux et le discours (figures, thèmes, motifs) varient. Cette alternance d'invariants et de variables permet de générer de l'intérêt chez les spectateurs, d'autant que l'hybridité série-feuilleton rend potentiellement instables tous les aspects de la matrice. Jason Mittell parle à ce sujet de complexité narrative ou de " complex TV» :

At its most basic level, narrative complexity redefines episodic forms under the influence of serial narration - not necessarily a complete merger of episodic and serial forms but a shifting balance. Rejecting the need for plot closure within every episode that typifies conventional episodic form, narrative complexity foregrounds ongoing stories across a range of genres. Complex television employs a range of serial techniques, with the underlying assumption that a series is a cumulative narrative that builds over time, rather than resetting back to a steady-state equilibrium at the end of every episode ${ }^{10}$.

\section{Les séries hybrides de l'imaginaire des années 1990 : The X-Files et Buffy}

Dans les premiers moments de la télévision, les séries pures, les feuilletons quotidiens et les séries anthologiques étaient les plus communes; les producteurs étaient convaincus que le public ne parviendrait pas à suivre une intrigue feuilletonnante complexe chaque semaine. Pour plusieurs spécialistes ${ }^{11}$, Hill Street Blues a tout changé en 1981 en « créant le genre de la série chorale et en démultipliant les possibilités de la série feuilletonesque ${ }^{12}$.» En 1987, Gene Roddenberry propose une suite à sa série culte: Star Trek: The Next Generation (CBS, 1987-1994), qui marque le début d'une télévision de plus en plus autoréflexive et autonome. Trois ans plus tard apparaît Twin Peaks de David Lynch et Mark Frost (ABC, 1990-1991), un feuilleton policier et fantastique proposant une complexité narrative bien plus grande que ce que la télévision offrait jusque-là, tout en jouant de l'humour et du surréalisme. La série ouvre la voie à plusieurs séries qui auront une importance culturelle et formelle majeure.

TV/Series, 15 | 2019 
11 The X-Files est créée par Chris Carter en 1993 (FOX) et s'étend sur neuf saisons, qui seront suivies de films et d'un retour sous la forme d'une minisérie à partir de 2016. En bref, The $X$-Files suit le travail de deux agents du FBI, qui parcourent tout le territoire américain pour enquêter sur des phénomènes étranges. Ils se retrouvent également au cœur d'un complot gouvernemental ancien visant à dissimuler au public la présence d'extraterrestres sur Terre et qui sera dévoilé très progressivement au fil des neuf saisons. La formule de la série emprunte donc à la fois au policier son double récit, crime et enquête ${ }^{13}$, au thriller politique le dévoilement très progressif d'un complot des puissants, au fantastique ses incertitudes épistémiques et son intérêt pour les différentes croyances, et à l'horreur ses effets de surprise, de grotesque, de répulsion, de monstration. Sur le plan de sa matrice, The X-Files est une série feuilletonnante composée de deux types d'épisodes. D'une part, ceux qui sont clos et racontent une enquête spécifique dans un lieu souvent unique - ce sont les épisodes stand-alone ou, comme les fans en sont venus à les nommer pour cette série, les épisodes "monster-of-the-week». D'autre part, les épisodes feuilletonnants, qui racontent la grande histoire de la conspiration, et sont souvent nommés "mytharc", qui correspond au macro-récit de la mythologie de la série. Ces derniers forment entre le quart et la moitié des épisodes de chaque saison, avec une augmentation au fil des années. Pour Alain Carrazé,

La série a contribué [...] à marquer définitivement le renouveau de la production des séries dramatiques. Avec sa narration partagée entre les loners et les épisodes de la mythologie, elle a contribué à rendre l'écriture plus sophistiquée, ouvrant encore plus la porte à des séries totalement feuilletonnantes, c'est-à-dire reposant sur un grand mystère central, comme le fera Lost ${ }^{14}$.

12 En 1997, quatre ans après le début de The X-Files, qui est alors au sommet de sa popularité, apparaît Buffy The Vampire Slayer, créée par Joss Whedon. Construite à la fois comme une inversion du stéréotype de la jeune fille blonde victime dans les films d'horreur, devenant elle-même ce que craignent les monstres, la série est aussi une métaphore sur le passage à l'âge adulte. La formule repose donc sur une hybridité générique importante : elle inverse le schéma actanciel de l'horreur et du gothique; emprunte à l'épopée sa quête, ses monstres, ses dieux et son héroïsme; au mélodrame sa trame familiale et amoureuse; au Bildungsroman son grand récit de passage à l'âge adulte; aux High School shows ses lieux, mais aussi certains de ses tropes; à la fantasy ses êtres surnaturels et ses pouvoirs magiques, etc. Du côté de la matrice, contrairement à The X-Files dans lequel le macrorécit se déploie sur toute la série, dans Buffy, chaque saison tourne autour d'un récit qui se clôt plus ou moins dans l'épisode final. Mais ce macro-récit saisonnier n'occupe pas l'ensemble des épisodes ou de l'espace narratif, une grande partie des épisodes sont clos et qualifiés de "monster-of-the-week", reprenant le terme inventé pour The X-Files. Contrairement à celle-ci, dans laquelle les épisodes sont structurés comme des romans policiers - avec le récit du crime dans le prologue et le récit de l'enquête dans les quatre actes qui suivent le générique -, dans Buffy, l'héroïne attend rarement qu'un crime survienne.

Ces séries hybrides amorcent un processus de complexification narrative qui s'accélère à la toute fin de la décennie, mais surtout au début du XXI ${ }^{\mathrm{e}}$ siècle, notamment avec les séries de HBO, puis de Showtime, FX ou AMC, aboutissant à un brouillage presque complet de la sérialité et de la feuilletonisation. De leur côté, les séries des années 1990 comme The $X$-Files ou Buffy portent encore les traces évidentes de ces formes; leur hybridation relève plutôt de la greffe que de l'amalgame et les sutures demeurent visibles ${ }^{15}$. Ainsi, on peut classer presque tous les épisodes comme relevant soit du feuilleton soit de la série, avec 
les quelques rares exceptions que sont les épisodes autonomes, et qui m'intéressent ici. Ces épisodes - leur grande popularité et l'investissement créatif et financier considérables qu'ils impliquent souvent - seraient donc les premiers signes de cette complexité narrative naissante. Mittell aborde d'ailleurs The X-Files et Buffy en ces termes :

The key prototypes for complex television emerged in the 1990s, setting precedents that more recent programs adopted and refined. The cult hit The X-Files exemplifies what may be the hallmark of narrative complexity: an interplay between the demands of episodic and serial storytelling, often oscillating between long-term arcs and stand-alone episodes. [...] According to many X- Files viewers and critics, the series suffered from too great a disjunction between the overly complex and endlessly deferred serial mythology and the detached independence of monster-ofthe-week episodes [...]. Buffy the Vampire Slayer and Angel are arguably more adept at juggling the dual demands of serial and episodic pleasures. [...] Within a given season, nearly every episode advances the season's arc while still offering episodic coherence and resolutions ${ }^{16}$.

\section{Les épisodes autonomes} classiques proposaient déjà des épisodes clos avec des structures narratives, stylistiques et thématiques originales et souvent métafictionnelles et intertextuelles. Pensons à certains épisodes de Star Trek: The Next Generation (en particulier les épisodes se déroulant sur le Holodeck ou impliquant le personnage de Q), par exemple. Ces épisodes autonomes presque complètement fermés, au sens où il y a résolution à la fin et qu'ils renferment peu de références aux autres épisodes de la série, s'inscrivent tout de même pour la plupart dans le macro-récit. Généralement, ils marquent une pause dans l'avancement de celui-ci, mais révèlent néanmoins des éléments de ce macro-récit déjà connus du public aux personnages, autrement pris dans trop de secrets, de trahisons, d'incapacités de communiquer, mais aussi dans leur rôle actanciel. Ce dévoilement n'est rendu possible que par un manque ou un décalage qui ouvre ainsi un espace narratif et psychologique. Les meilleurs exemples se trouvent sans doute dans Buffy: l'héroïne n'arrive à révéler à ses amis qu'elle était au paradis que dans un épisode musical (" Once More With Feeling », S06E07), elle ne parvient à révéler sa véritable identité à son petit copain Riley que dans un épisode sans parole («Hush», S04E10) et Tara n'apprend que son esprit est manipulé par Willow et sa magie que lorsque tous les personnages oublient leur identité (« Tabula Rasa » S06E08). L'innovation formelle joue donc un rôle narratif en soi. Tous ces épisodes, souvent réussis et très populaires ${ }^{17}$, proposent de réfléchir à la narrativité des séries télévisées : comment raconte-t-on en séries sans se répéter, en innovant, en disant quelque chose de neuf des personnages après de très nombreuses saisons et des dizaines d'heures de récit audiovisuel? La réponse semble être: en variant les narrateurs, les structures, les cadres diégétiques poreux ou redessinés, ainsi qu'avec des métalepses, des vitesses de narration variables, des mondes parallèles comme source narrative, etc. Ces procédés peuvent être classés en trois grandes catégories, sur lesquelles je reviendrai plus en détail : la fictionnalité, la narrativité et la transtextualité.

Dans The X-Files, on peut identifier seize épisodes répartis sur les neuf saisons (voir la liste en annexe), mais surtout concentrés sur les saisons quatre à sept, ce qui n'est pas si surprenant. Dans la section « Format-bending and meta episodes» de son article sur The $X$-Files, Juliette Harrisson l'explique bien : 
For its first few years [...], the format of the show remained broadly stable. However, as the years went by, the creative team became more and more willing to play around with that format, producing a variety of weird, sometimes very meta episodes [...]. In order to bend a show's format, you have to have an established format in the first place, so there aren't any particularly notable format-bending episodes in Seasons One and Two ${ }^{18}$.

Parmi ces épisodes autonomes, seulement deux sont écrits et réalisés par Chris Carter, qui a surtout tendance à s'intéresser aux épisodes mythologiques (feuilletonnants). Ces deux épisodes, «The Post-Modern Prometheus» (S05E05) et «Triangle» (S06E03), sont toutefois parmi les plus intéressants, notamment sur le plan formel, ce dernier proposant un thriller d'espionnage nazi en mer en adoptant des plans-séquences et des splits screens spectaculaires. Vince Gilligan, désormais plus célèbre pour sa propre série Breaking Bad (AMC, 2008-2013), est responsable du scénario de sept de ces épisodes, et David Duchovny, de deux d'entre eux. La plupart de ces épisodes jouent sur les points de vue, la matérialité de l'image, le rapport à la fiction, à la vérité et au témoignage. Mentionnons notamment un épisode singulier qui tente une expérimentation formelle spectaculaire : "Redrum » (S08E06) propose un temps narratif et discursif complexe, puisque tous les personnages évoluent chronologiquement à l'exception du personnage source de la focalisation narrative qui progresse anté-chronologiquement, avec le spectateur.

De son côté, «Buffy is probably the most accomplished series for narratively spectacular theme episodes, with individual episodes predicated on narrative devices such as starkly limiting storytelling parameters [...], genre mixing [...], shifts in perspective [...], or foregrounding an unusual narrator $[. . .]^{19}$. » Dans la série, on peut identifier dix-neuf de ces épisodes qui rompent, plus ou moins radicalement selon les cas, avec la matrice de la série, situés surtout dans les saisons trois à six, pour les mêmes raisons que The X-Files. Alors que Joss Whedon écrit et réalise moins de $15 \%$ de tous les épisodes, il dirige plus de $30 \%$ des épisodes autonomes. Les épisodes qui mettent surtout en avant des procédés narratifs particulièrement originaux sont assez peu nombreux, mentionnons néanmoins le très réussi «Conversations with Dead People ", qui isole les personnages principaux les uns des autres et présente, en temps réel, des discussions qu'ils ont avec des personnages plus ou moins secondaires morts dans la série, ou encore «Storyteller » (S07E16), entièrement raconté du point de vue d'un personnage secondaire, Andrew, à la fois mauvais lecteur de la série et narrateur non fiable. Aussi intéressantes que soient ces explorations formelles, il n'en demeure pas moins que les épisodes qui interrogent de manière originale la fictionnalité et la réalité des récits et des mondes perçus dominent largement la série Buffy. Parmi les épisodes qui brouillent les frontières entre les rêves, l'inconscient et la réalité, "Normal Again » (S06E17) va d'ailleurs jusqu'à remettre en question l'existence même du monde fictionnel de la série, alors que dans de nombreux épisodes, c'est la magie qui altère la réalité des personnages. Un autre épisode qui se distingue nettement est "The Body » (S05E16), un épisode primé qui décrit les sentiments contradictoires d'engourdissement et d'hypersensibilité, mais aussi d'allongement et de raccourcissement temporel, après avoir découvert le cadavre d'un proche. Le réalisme de cet épisode détonne avec le ton fantastique habituel de la série, créant une véritable rupture fictionnelle et remettant en question par le fait même la perception du monde des personnages et du spectateur. 


\section{Typologie des épisodes autonomes}

Selon Danielle Aubry,

la structuration mnémonique de la pensée et de l'expression [...] pourrait très bien définir, avec l'avènement de médias audiovisuels, une opération métatextuelle qui aurait pour fonction de faire circuler des archétypes narratifs (structurés par les genres) d'un médium à l'autre ou encore contribuer à une espèce de banque de motifs narratifs (qu'ils deviennent des stéréotypes ou non) à l'intérieur d'un même médium ${ }^{20}$.

En étudiant ce corpus d'épisodes, il est possible d'identifier un certain nombre de tropes (ou motifs), que l'on peut ainsi regrouper en une typologie des écarts à la matrice sérielle des épisodes autonomes en fonction de la fictionnalité, de la narrativité et de la transtextualitéé ${ }^{21}$. Dans la fictionnalité, il y a les distorsions temporelles (boucles, inversions, retours en arrière, accélérations et ralentissements), les doubles et les mondes parallèles (version altérée de la réalité habituelle de la série) et les mondes fictionnels issus de rêves ou de l'inconscient. Ensuite, dans la catégorie de la narrativité, se trouvent les récits à points de vue multiples et les narrateurs non fiables, d'une part, et les métalepses et narrateurs ou personnages omnipotents, d'autre part. Finalement, la transtextualité comprend l'intertextualité (pastiches, hommages, citations) et l'architextualité (ruptures ou hybridités génériques). Dans The X-Files, il y a une surreprésentation des catégories " points de vue multiples et narrateurs non fiables ", de même que celles de l'« intertextualité » (surtout des pastiches) et de l'« architextualité " (hybridité générique). Dans Buffy, c'est plutôt la fictionnalité, avec les doubles et les mondes parallèles et, pour la narrativité, les personnages omnipotents qui créent ou altèrent le récit, qui dominent.

\section{Épisodes autonomes et fictionnalité}

Du côté de la fictionnalité, le cas le plus commun de distorsion temporelle est la boucle (ou time loops, ou plus couramment Groundhog Day Loops, en référence au film du même nom, réalisé par Harold Ramis en 1993), qui marque un manque de continuité temporelle, de chronologie. Il s'agit d'épisodes dans lesquels un ou des personnages se retrouvent à vivre sans fin la même journée ou la même séquence temporelle plus ou moins longue jusqu'à apprendre une leçon ou trouver une solution à l'énigme. Parfois les personnages sont conscients de cette boucle dès le début, parfois ils n'en prennent conscience qu'après de très nombreuses occurrences, à cause d'impressions de « déjà vu » ou grâce à d'autres personnages. Cette distorsion du temps du récit provoque aussi une distorsion du temps narratif, qui est également pris dans une boucle. Cet exercice de style permet surtout d'explorer les possibles de la fiction, à l'image des " jardins aux sentiers qui bifurquent ${ }^{22}$ " de Jorge Luis Borges, et il met en abyme la sérialité elle-même. Il permet d'épuiser les possibles fictionnels en explorant toutes les issues à une intrigue, mais aussi de placer l'un des personnages principaux dans une situation impossible sans aide extérieure, le forçant à expérimenter, lui aussi, avec les possibles pour trouver une issue, tout en lui donnant une certaine liberté à l'endroit des autres personnages qu'il n'a pas habituellement. Puisque la boucle recommencera, il n'y a a priori aucune conséquence aux actes commis, ce qui pousse souvent les personnages (et les scénaristes) à explorer des relations décalées par rapport à la matrice de la série (relation amoureuse, relation entre 
employé et employeur, entre client et vendeur, etc.) et à explorer un aspect inhabituel de la vie ou de la personnalité du personnage (humour, quotidien, impatience, etc.). L'autre aspect formel qui résulte de ce trope est la nécessité d'une grande inventivité dans la mise en scène et les angles de caméra, afin d'éviter la monotonie. Chaque répétition de la boucle devient ainsi un jeu des erreurs pour le spectateur.

On peut en trouver des exemples ${ }^{23}$ dans Star Trek: The Next Generation et Star Trek: Discovery (CBS, 2017-présent), Stargate SG1 (Showtime > Sci Fi, 1997-2007), Supernatural (The CW, 2005-présent), Fringe (FOX, 2008-2013), Person of Interest (CBS, 2001-2016), Dark Matter (Space et Syfy, 2015-2017), etc. Dans les deux séries qui nous intéressent, il s'agit des épisodes "Monday» (S06E14) dans The X-Files et «Life Serial» (S06E05) dans Buffy. Le premier place Mulder dans une boucle de la pire journée de sa vie (inondation, retard au travail, braquage de banque), mais c'est un personnage secondaire, la petite amie du braqueur, qui est en fait la victime et le témoin de la boucle. À chaque répétition, elle tente de retenir son partenaire, par la parole ou les actes, contacte le FBI, puis Mulder et Scully directement. Chaque jour, elle observe la banque exploser. C'est par une impression de « déjà vu » que Mulder finit par comprendre et permettre à la femme de briser la boucle en entrant elle-même dans la banque, mais se faisant ainsi tuer par accident. Cet épisode nous donne accès au quotidien de Mulder, son rapport aux objets, à son appartement, aux tâches de la vie, mais surtout il permet d'exposer la souffrance banale et aliénante d'une femme dans une situation abusive et répétitive, incapable de trouver la solution malgré tous ses efforts et ses appels à l'aide. Contrairement à d'autres exemples de ce type, aucun apprentissage n'est fait, personne n'en ressort grandi. Dans l'épisode de Buffy, il s'agit d'un des quatre actes de l'épisode, dans lequel Buffy cherche une carrière après avoir ressuscité quelques semaines plus tôt. Elle tente donc de devenir vendeuse dans la boutique de magie de ses amis, mais quelqu'un lui jette un sort qui la place dans une boucle temporelle, la forçant à répéter à l'infini une séquence de quelques minutes tant qu'une cliente qui veut une chose impossible n'est pas satisfaite. Comme c'est souvent le cas, cet épisode joue le trope sur le mode humoristique, critiquant au passage l'aliénation du travail répétitif, les relations humaines absurdes dans un contexte capitaliste, mais aussi la nécessité de l'inventivité et de l'imagination pour le personnage qui veut s'échapper et pour les scénaristes qui veulent épuiser les possibles.

Du côté des doubles et des mondes parallèles, mais aussi des rêves et de l'inconscient, la rupture avec la matrice sérielle peut-être plus ou moins importante et subtile. Certaines séries, notamment Buffy, comprennent beaucoup d'exemples de ce type de décalage, qui peut aller d'un changement de personnalité plus ou moins radical de tous les personnages à cause de sortilèges quelconques («Halloween » [S02E06], « Something Blue » [S04E09], « Band Candy » [S03E06], « Superstar » [S04E17]), à une altération complète du tissu de la réalité ou de ses règles (« Normal Again » [S06E17], « Nightmares » [S01E10], « Restless » [S04E22]). Malgré la rupture qu'ils opèrent, les manques (de normalité, de sommeil, de raison, de rationalité, etc.) vers lesquels ils pointent, ces mondes dédoublés nous rappellent la nature même de la fiction et des mondes parallèles infinis qu'elle génère ${ }^{24}$. Si ce sont des épisodes clos, un spectateur qui ne connait pas la matrice sérielle ne pourra en comprendre les enjeux ni les apprécier : ces épisodes ne sont intéressants que par l'écart qu'ils opèrent (par exemple, on ne peut comprendre l'étrangeté d'un vampire Willow ou d'un Jonathan superstar, si on ne connait pas ces personnages), montrant bien qu'ils ne sont pas du tout autonomes, malgré les apparences. D'ailleurs, Joss Whedon 
s'amuse à intégrer dans ces épisodes des indices sur l'intrigue des prochaines saisons, qui ne pourront être compris qu'a posteriori.

\section{Épisodes autonomes et narrativité}

Du côté de la narrativité, la question des narrateurs non fiables (manque de fiabilité) et de la multiplication des points de vue narratifs (manque de cohérence) est intéressante dans The X-Files, puisqu'elle répond thématiquement et idéologiquement à toutes les conspirations, les complots, les témoignages douteux (et doutés) sur des phénomènes inexpliqués mis en scène. Le cas de « Musings of A Cigarette Smoking Man » (S04E07) est notable. Dans le prologue, on y voit l'homme à la cigarette qui espionne les Lone Gunmen (des informateurs de Mulder adeptes des théories du complot) et les écoute raconter sa propre histoire. Puis, chaque acte est consacré à une époque et un événement (les assassinats de John F. Kennedy et de Martin Luther King, l'autopsie de l'extra-terrestre de Roswell), dans un style différent. Or, rien n'indique que ces fragments, à la facture a priori réaliste, ne sont pas fiables. Il faut se souvenir qu'ils sont racontés par des adeptes du complot et correspondent sans doute aux fantasmes des trois hommes. D'ailleurs, l'épisode se termine sur l'homme à la cigarette qui décide de ne pas les assassiner, ce qui indique sans doute qu'ils sont assez loin de la réalité.

L'autre cas intéressant est celui des épisodes adoptant le style «Rashomon » (nommé à partir du film de Kurosawa et désignant un récit raconté par plusieurs narrateurs non fiables qui se contredisent). C'est le cas de l'épisode "El Mundo Gira (S04E11), qui s'amuse à mettre en images de multiples versions de la même histoire sur la base d'un savoir narratif, mais surtout des croyances et du folklore, chacun y voyant une attaque chimique ou El Chupacabra, des extraterrestres ou des agents du FBI en combinaison. L'épisode «Bad Blood» (S05E12) propose une autre version très amusante et représentative des tensions et des différences de points de vue entre Mulder et Scully. Le prologue nous montre Mulder traquant un homme et le tuant avec un pieu, avant de découvrir qu'il portait de fausses dents de vampire. En attendant le moment fatidique où Mulder devra faire son rapport, il demande à Scully de raconter l'histoire de son point de vue, puis il fait de même, tous les deux adoptant une mauvaise foi assez évidente. Leurs travers sont exagérés jusqu'à la caricature ; l'un apparaît la victime innocente de l'autre ; le physique des personnages secondaires diffère, etc. C'est une critique de la valeur des témoignages, qui sont toujours problématiques dans The X-Files, autant que de la réalité qu'ils tentent de décrire, de la personnalité des personnages et de l'état de leurs relations à ce point de la série.

\section{Épisodes autonomes et transtextualité}

Finalement, la transtextualité offre également l'occasion d'épisodes originaux qui ont recours à différents tropes largement décrits par Gérard Genette tels que le pastiche, l'hommage, la citation ou l'architextualité (transtextualité générique, au sens de Genette). Les œuvres évoquées peuvent être littéraires, cinématographiques ou télévisuelles, mais il peut aussi s'agir de genres, plus largement. La simple référence générique permet ainsi à ces épisodes autonomes d'adopter les codes de genres éloignés de la série dans son ensemble et clairement identifiables. Ainsi, des séries fantastiques comme Buffy et The XFiles proposent des épisodes de cinéma muet («Hush», Buffy, S04E10), de comédie

TV/Series, 15 | 2019 
musicale («Once More With Feeling» Buffy, S06E07) ou de road movie («Drive», The XFiles, S06E02). Ces épisodes ne citent aucune œuvre en particulier appartenant à ces genres, ou plutôt ils s'amusent à les évoquer toutes à travers l'atmosphère, l'esthétique, les tropes, la structure, etc.

Puis, il y a certains cas particulièrement intéressants (et souvent postmodernes) qui ne font pas référence à un genre ou à une œuvre unique, mais qui s'inscrivent plutôt dans un réseau d'œuvres déjà transmédiatique. C'est le cas des épisodes évoquant Frankenstein, autant le roman de Mary Shelley que ses différentes adaptations : ainsi l'épisode de The XFiles « The Post-Modern Prometheus » rend un hommage visuel au film de James Whale de 1931, alors que Buffy en offre une version recadrée dans la culture très américaine du football adolescent et rejoue le drame familial en déplaçant les enjeux. À l'inverse de Carter, Whedon, dans "Some Assembly Required» (S02E02) tient pour acquis que l'influence de Frankenstein sur la culture populaire est telle qu'il est superflu de citer directement l'œuvre originale, offrant plutôt un clin d'œil. Par contre, il adopte le pastiche et les références explicites lorsqu'il aborde le roman de Bram Stoker et ses nombreuses adaptations dans «Buffy vs Dracula » (S05E01). Mentionnons aussi, dans The X-Files, l'épisode « Ice » (S01E08), qui pastiche le film From Another World (Christian Nyby, 1951) et son remake The Thing (John Carpenter, 1982).

Enfin, le cas de la transtextualité plus spécifiquement télésérielle est sans doute le plus révélateur dans le contexte des épisodes autonomes, puisqu'il met de l'avant l'autonomisation de cet art et use de tropes qui lui sont plus spécifiques. Le développement de la série télévisée au sein des différentes chaînes et avec des équipes communes de production a ainsi mené à l'apparition d'épisodes cross-over. Dans Buffy, il s'agit des épisodes au cours desquels les personnages du spin-off Angel reviennent à Sunnydale, mais ils sont rarement autonomes, étant donné leur rôle dans la mythologie de la série. The X-Files offre un exemple plus probant avec « Unusual Suspect » (S05E03), un cross-over avec la série Homicide, et deux cas d'intertexte particulièrement réussi : « XCops » (S07E12), qui va très loin sur le plan formel dans son pastiche de l'émission Cops et «Sunshine Days » (S09E18), qui cite abondamment The Brady Bunch.

La plupart des tropes présentés ici, notamment le style "Rashomon", les boucles temporelles et les pastiches sont très communs et apparaissent sous une forme ou un autre dans de nombreuses séries qui sont pourtant de genres très différents, du sitcom au feuilleton de science-fiction. Ils montrent que la sérialité de ces épisodes se joue dans les codes génériques, dans la répétition des séries entre elles, une forme d'exercice de style obligé qui relève de l'architexte du grand genre de la série télévisée.

\section{Le genre, les écarts, le plaisir et la sérialité}

Ces épisodes autonomes sont souvent décrits comme étant des moments où les créateurs de série s'amusent et expérimentent au-delà des contraintes imposées par la production d'une série télévisée populaire. Ce sont des moments de liberté formelle et narrative qu'ils se permettent. Par exemple, à propos de l'épisode «The Post-Modern Prometheus », la blogueuse Emily VanDerWerff écrit : "Another from Carter, this time playing around with the show's film style and format. This was something the series did more and more often in its later seasons, and this is one of the first experiments ${ }^{25}$. » On pourrait en déduire que c'est parce que ces épisodes sont relativement rares qu'ils sont 
possibles. À propos de l'épisode «Triangle » et des plans-séquence, Chris Carter confiait au journaliste Alain Carrazé : "On avait fait cela pour la saison six à une époque où on avait gagné le droit de faire ce genre d'expérimentation. Ça a été un travail de titan ${ }^{26}$. $» \mathrm{Et}$ d'ailleurs, c'est parce qu'il représente une rupture qu'ils sont intéressants : une rupture dans une itération, dans une matrice assez souvent répétée pour devenir familière, autant pour les créateurs, que pour le public. Ce sont des événements qui brisent le quotidien et qui ne sont possibles que dans les séries qui présentent un grand nombre d'épisodes par année. C'est parce que le public est déjà fan qu'il est prêt à suivre et qu'il pourra goûter la rupture, apprécier la folie ou le décalage. En effet, Mittell explique que l'originalité et la complexité de ces épisodes autonomes encouragent un jeu de décodage formel chez les spectateurs :

Through the operational aesthetic, these complex narratives invite viewers to engage at the level of formal analyst, dissecting the techniques used to convey spectacular displays of storytelling craft; this mode of formally aware viewing is highly encouraged by these programs, as their pleasures are embedded in a level of awareness that transcends the traditional focus on diegetic action that is typical of most mainstream popular narratives ${ }^{27}$.

Paradoxalement, si ces épisodes brisent la matrice des séries dans lesquelles ils sont insérés (matrice qui en fait normalement le succès), ils sont souvent considérés comme les meilleurs épisodes par les fans, les critiques ${ }^{28}$, les jurys ${ }^{29}$ et les créateurs eux-mêmes ${ }^{30}$. C'est le grand paradoxe : on aime les séries télévisées en partie à cause de leur sérialité, du sentiment de familiarité formelle que celle-ci procure, mais on aime encore plus lorsqu'elles en sortent, à l'occasion. Monica Michlin se pose d'ailleurs la question : «In a double-bind, how does contemporary TV fiction avoid the pitfalls of too-obvious seriality how does it disrupt linear progression, avoid repetition, renew its esthetics of surprise? ${ }^{31}$ » Pour Vincent Jouve, qui s'arrête à la notion de séduction narrative avant de l'appliquer aux séries, l'intérêt que suscite un récit chez le lecteur dépend de l'inattendu (ou intérêt narratif), de la complexité (intérêt herméneutique) et de l'intensité émotionnelle (surprise, suspense et curiosité, causée par la proximité, l'improbabilité et la gradualité). Il ajoute que les séries, contrairement aux récits clos en général, ont la spécificité de permettre une grande familiarité avec les personnages, de se dérouler dans un temps non-limité et d'être faciles d'accès.

31 Les épisodes autonomes se retrouvent donc avec un statut hybride. La série fournit la familiarité, mais l'autonomie de l'épisode apporte de la complexité et un temps narratif clos, tout en provoquant un certain effet d'inattendu sériel et une discrimination (considérée comme écart à la norme, aux habitudes du spectateur). Et puisque la télévision se construit (historiquement et formellement) si près de la vie quotidienne des spectateurs (qui la consomment dans leur foyer jour après jour), cette fascination s'explique: il s'agit d'un événement, d'une rupture de la répétition du quotidien des spectateurs eux-mêmes. À propos de la série Mission Impossible, Esquenazi écrit :

La réussite narrative de la série est constitutivement attachée à cette ritualité sérielle : elle est absolument nécessaire à la mise en valeur des enjeux de chacun des épisodes. Car la régularité et même la platitude y ont une fonction très particulière : elles autorisent les extravagances de l'accomplissement des missions ${ }^{32}$

Ce qui nous ramène au plaisir générique créé par les attentes d'un genre et au passage au second degré selon Genette, ce « moment où l'observance, nécessairement consciente, de la convention se nuance d'un autopastiche plus ou moins ironique ", ce qui peut donner le 
« sentiment d'une transgression des normes, transgression qui les conforte en révélant à temps une fausse transgression ${ }^{33}$.»

\section{Conclusion}

33 L'émergence de la complexité narrative dans certaines séries télévisées des deux dernières décennies ne s'est pas produite $d u$ jour au lendemain et la production d'épisodes autonomes au sein de séries hybrides dans les années 1990 a très certainement fait partie de ce processus. Bien sûr, au-delà de leur originalité évidente, l'autonomie de ces épisodes est loin d'aller de soi et leur étude tend à montrer qu'ils s'insèrent malgré tout dans des matrices sérielles et jouent un grand rôle dans la structure des séries, dans l'esthétique qu'elles privilégient, dans le savoir narratif et générique qu'elles portent, dans leur interdiscursivité, ne serait-ce qu'en offrant aux créateurs et aux spectateurs un contraste, une distance, un temps d'arrêt. Mais il ne faut pas oublier la mythologie qu'elles contribuent malgré tout à mettre en place. Florent Favard nomme d'ailleurs ces épisodes des «mythalones ", qu'il décrit ainsi :

Même les épisodes les plus «indépendants » peuvent avoir une influence sur la mythologie et ses arcs narratifs majeurs : [...] une mise à distance visant à reconsidérer la mythologie sous un angle inédit. Le recul que les épisodes «bouclés » peuvent apporter aux arcs narratifs majeurs d'une série témoigne d'une fluidité qui, depuis The X-Files, a contaminé nombre de séries narrativement complexes $^{34}$.

Mais si les épisodes autonomes s'éloignent des matrices des séries dont elles font partie et offrent un univers esthétique et formel relativement clos, ne peut-on pas établir un lien avec les séries anthologiques des débuts de la télévision, comme Twilight Zone ou Alfred Hitchcock Presents? Et ce d'autant plus qu'ils s'inscrivent bien souvent dans les mêmes genres de l'imaginaire comme l'horreur ou la science-fiction. Le format anthologique prévoit bien sûr des épisodes clos, par définition, mais est-ce vraiment à dire que ces séries sont complètement dénuées de matrice? Bien sûr que non; si celle-ci n'est pas narrative, elle est plutôt esthétique et thématique. Ainsi, les épisodes autonomes s'en rapprochent bien davantage que du cinéma, par exemple.

On peut également se demander si la plus grande complexité narrative qui caractériserait de nombreuses séries contemporaines selon Jason Mittell permet encore l'existence d'épisodes autonomes, ou bien s'il s'agissait d'une particularité spécifique aux séries hybrides qui ont marqué le début de ce processus dans les années 1990 ? Y a-t-il encore une matrice claire de laquelle s'éloigner? Il est sans doute en effet plus difficile d'identifier aujourd'hui de tels épisodes, puisque l'originalité et la diversité formelles caractérisent justement les séries dans leur entièreté, mais il n'en demeure pas moins que l'on peut toujours observer, même dans les séries les plus complexes, des épisodes qui se démarquent, qui offrent des ruptures. C'est par exemple le cas dans Westworld (HBO, 2016présent) avec son épisode "Kiksuya » (S02E08), presque entièrement en langue lakota ; dans Twin Peaks (ABC > Showtime, 1990-1991, 2017) avec «Gotta Light?» (S03E08), épisode déjà célèbre qui relève davantage du cinéma d'avant-garde que de la télévision grand public ; The Good Place (NBC, 2016-présent), dont tous les personnages sont joués par la même actrice dans l'épisode «Janet(s)» (S03E10); ou encore le « Chapter 7 » (S01E07) de Legion (FX, 2017-présent) et son hommage au cinéma expressionniste allemand. On pourrait même se questionner sur le statut de "Bandersnatch» (2018), le film (ou 
épisode ?) interactif « hors-série » de Black Mirror (Channel 4 > Netflix, 2011-présent). Il semble que la volonté de s'éloigner à l'occasion des habitudes matricielles, de jouer avec les possibles narratifs de son univers de fiction, les limites de ses personnages ou de son esthétique, continue d'inspirer les créateurs dans leur besoin d'innovation télésérielle.

\section{BIBLIOGRAPHIE}

«"Groundhog Day" Loop », TV Tropes, consultée le 20 mai 2018 : http://tvtropes.org/pmwiki/ pmwiki.php/Main/GroundhogDayLoop.

AUBRY, Danielle, Du roman-feuilleton à la série télévisuelle. Pour une rhétorique du genre et de la sérialité, Berne, Peter Lang, 2006.

AUDET, René, GEFEN, Alexandre (dir.), Frontières de la fiction, Québec et Bordeaux, Nota Bene et Presses Universitaires de Bordeaux, 2001.

BARONI, Raphaël, MACÉ, Marielle, « Avant-propos », in BARONI, Raphaël, MACÉ, Marielle (dir.), Le savoir des genres, Rennes, PUR, 2007, p. 7-17.

BENASSI, Stéphane, Séries et feuilletons T.V. : Pour une typologie des fictions télévisuelles, Liège, Éditions du CÉFAL, 2000.

BENASSI, Stéphane, « Sérialité(s) et esthétique de la fiction télévisuelle », Belphégor, Volume 14, n ${ }^{\circ} 1,2016$, https://journals.openedition.org/belphegor/770?lang=en

BORGES, Jorge Luis, « Le jardin aux sentiers qui bifurquent », in Fictions, Paris, Gallimard, (1941) 1983, p. 91-104.

CARRAZÉ, Alain, NIGITA, Romain, Les nouveaux feuilletonistes : Ils ont révolutionné les séries TV, Paris, Fantask, 2016.

CORNILLON, Claire, « La forme semi-feuilletonnante formulaire : l'exemple d'Ally McBeal », TV/ Series, $n^{\circ} 15,2019$.

ESQUENAZI, Jean-Pierre, Les séries télévisées : l'avenir du cinéma ?, 2 édition, Paris, Armand Colin, 2014.

FAVARD, Florent, Écrire une série TV : la promesse d'un dénouement, Tours, Presses universitaires François Rabelais, 2019.

GENETTE, Gérard, Discours du récit, Paris, Seuil, 2007.

GENETTE, Gérard, Fiction et diction, Paris, Seuil, 1991.

GENETTE, Gérard, Figures V, Paris, Seuil, 2002.

GENETTE, Gérard, Palimpsestes : la littérature au second degré, Paris, Seuil, 1982.

HARRISSON, Juliette, « The X-Files: A Binge Guide for Beginners », Den of Geek !, 28 décembre 2017 : http://www.denofgeek.com/us/tv/the-x-files/244152/the-x-files-a-binge-guide-forbeginners 
JOUVE, Vincent, « Le plaisir sériel », in Pop-en-stock. Séries télé : saison un, Montréal, Éditions de ta mère, coll. « Essais », 2014, p. 15-34.

LAVOCAT, Françoise (dir.), La théorie littéraire des mondes possibles, Paris, CNRS Éditions, 2010.

MACÉ, Marielle, Le genre littéraire, Paris, GF Flammarion, coll. « Corpus ».

MICHLIN, Monica, « More, More, More: Contemporary American TV Series and the Attractions and Challenges of Serialization as Ongoing Narrative », Mise au point, no 3, 2011 : https:// journals.openedition.org/map/927.

MITTELL, Jason, Complex TV. The Poetics of Contemporary Televion Storytelling, New York, NYU Press, 2015.

SOULEZ, Guillaume, «La double répétition : structure et matrice des séries télévisées », Mise au point, $n^{\circ} 2,2011$ : https://journals.openedition.org/map/979\#ftn3.

THOMPSON, Robert, Television's Second Golden Age: From Hill Street Blues to ER, Syracuse (NY), Syracuse University Press, 1997.

TODOROV, Tzetan, « Typologie du roman policier », in Poétique de la prose, Paris, Seuil, 1971, p. $55-65$.

VANDERWERFF, Emily, «10 Must-See Episodes of The X-Files », AV/TV Club, 20 juillet 2012 : https://tv.avclub.com/10-must-see-episodes-of-the-X-files-1798232355.

\section{ANNEXES}

\section{Annexe : Épisodes autonomes qui s'écartent de la matrice sérielle}

The X-Files (Chris Carter, FOX, 1993-2002)

\begin{tabular}{|c|c|c|c|c|}
\hline $\begin{array}{l}\text { Saison/ } \\
\text { épisode }\end{array}$ & $\begin{array}{l}\text { Titre de } \\
\text { l'épisode }\end{array}$ & $\begin{array}{l}\text { Réalisateur; } \\
\text { Scénariste(s) }\end{array}$ & Résumé & Tropes \\
\hline 1.8 & Ice & $\begin{array}{ll}\text { David } & \text { Nutter ; } \\
\text { Glen } & \text { Morgan } \\
\text { et James Wong }\end{array}$ & $\begin{array}{l}\text { Un microorganisme } \\
\text { trouvé dans la glace sur } \\
\text { une base arctique rend } \\
\text { violente et paranoïaque } \\
\text { l'équipe de scientifiques. }\end{array}$ & $\begin{array}{l}\text { Transtextualité } \\
\text { intertextualité } \\
\text { (hommage, pastiche) }\end{array}$ \\
\hline 3.20 & \begin{tabular}{|lr} 
Jose & Chung's \\
From & Outer \\
Space &
\end{tabular} & $\begin{array}{l}\text { Rob Bowman; } \\
\text { Darin Morgan }\end{array}$ & $\begin{array}{l}\text { Un auteur veut écrire un } \\
\text { roman de science-fiction } \\
\text { non fictif à propos d'un } \\
\text { enlèvement sur lequel } \\
\text { Mulder et Scully ont } \\
\text { enquêté. Scully lui } \\
\text { raconte. }\end{array}$ & $\begin{array}{l}\text { Narrativité / points de } \\
\text { vue multiples et } \\
\text { narrateurs non fiables; } \\
\text { métalepse; } \\
\text { Transtextualité/ } \\
\text { intertextualité (pastiche) }\end{array}$ \\
\hline
\end{tabular}




\begin{tabular}{|c|c|c|c|c|}
\hline 4.7 & $\begin{array}{l}\text { Musings of A } \\
\text { Cigarette } \\
\text { Smoking- } \\
\text { Man }\end{array}$ & $\begin{array}{l}\text { James Wong; } \\
\text { Glen Morgan }\end{array}$ & $\begin{array}{l}\text { Le Cigarette } \text { Smoking } \\
\text { Man écoute les } \\
\text { Gunmen raconter } \\
\text { propre vie d'assassinats } \\
\text { historiques } \\
\text { conspirations. }\end{array}$ & $\begin{array}{l}\text { Narrativité / points de } \\
\text { vue multiples et } \\
\text { narrateurs non fiables; } \\
\text { personnage omnipotent; } \\
\text { Transtextualité / } \\
\begin{array}{l}\text { architextualité } \\
\text { (historique, thriller } \\
\text { politique) }\end{array}\end{array}$ \\
\hline 4.11 & $\begin{array}{ll}\text { El } & \text { Mundo } \\
\text { Gira } & \end{array}$ & $\begin{array}{l}\text { Tucker Gates; } \\
\text { John Shiban }\end{array}$ & $\begin{array}{l}\text { Une femme raconte } \\
\text { l'histoire d'une attaque } \\
\text { d'El chupacabra, avant } \\
\text { d'être contredite. }\end{array}$ & $\begin{array}{l}\text { Narrativité / points de } \\
\text { vue multiples et } \\
\text { narrateurs non fiables; } \\
\text { Transtextualité / } \\
\text { architextualité (telenovela, } \\
\text { conte oral) }\end{array}$ \\
\hline 5.3 & $\begin{array}{l}\text { Unusual } \\
\text { Suspect }\end{array}$ & $\begin{array}{l}\text { Kim Manners ; } \\
\text { Vince Gilligan }\end{array}$ & $\begin{array}{l}\text { Épisode flashback de } \\
\text { rencontre entre Mulder } \\
\text { et les Lone Gunmen ( } \\
\text { crossover avec la série } \\
\text { Homicide). }\end{array}$ & $\begin{array}{l}\text { Transtextualité / } \\
\text { intertextualité (citation); } \\
\text { Fictionnalité / distorsion } \\
\text { temporelle }\end{array}$ \\
\hline 5.5 & $\begin{array}{l}\text { The Post- } \\
\text { Modern } \\
\text { Prometheus }\end{array}$ & $\begin{array}{l}\text { Chris Carter; } \\
\text { Chris Carter }\end{array}$ & $\begin{array}{l}\text { Un savant fou crée un } \\
\text { monstre qu'il } \\
\text { abandonne; son père } \\
\text { agriculteur s'en occupe et } \\
\text { tente de lui fabriquer une } \\
\text { compagne, à l'image de } \\
\text { Frankenstein. }\end{array}$ & $\begin{array}{l}\text { Transtextualité / } \\
\text { intertextualité } \\
\text { (hommage, pastiche) }\end{array}$ \\
\hline 5.12 & Bad Blood & $\begin{array}{lr}\text { Cliff } & \text { Bole } \\
\text { Vince } & \text { Gilligan }\end{array}$ & $\begin{array}{l}\text { Mulder tue un supposé } \\
\text { vampire; Mulder et } \\
\text { Scully racontent chacun } \\
\text { leur perception très } \\
\text { différenter } \\
\text { événements. }\end{array}$ & $\begin{array}{ll}\text { Narrativité / points de } \\
\text { vue multiples et } \\
\text { narrateurs non fiables }\end{array}$ \\
\hline 6.2 & Drive & $\begin{array}{l}\text { Rob } \text { Bowman ; } \\
\text { Vince }\end{array}$ & $\begin{array}{l}\text { Mulder aide un homme à } \\
\text { rouler très vite vers } \\
\text { l'ouest pour éviter que sa } \\
\text { tête n'explose. }\end{array}$ & $\begin{array}{l}\text { Transtextualité / } \\
\text { architextualité (road } \\
\text { movie) }\end{array}$ \\
\hline 6.3 & Triangle & $\begin{array}{l}\text { Chris Carter } \\
\text { Chris Carter }\end{array}$ & 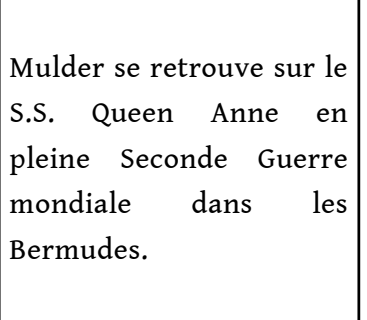 & $\begin{array}{l}\text { Fictionnalité / distorsion } \\
\text { temporelle; doubles et } \\
\text { mondes parallèles ; rêves ; } \\
\text { Transtextualité / } \\
\text { architextualité } \\
\text { (historique, film } \\
\text { d'espionnage) }\end{array}$ \\
\hline
\end{tabular}




\begin{tabular}{|c|c|c|c|c|}
\hline 6.14 & Monday & $\begin{array}{l}\text { Kim Manners } \\
\text { Vince Gilligan } \\
\text { et John Shiban }\end{array}$ & $\begin{array}{l}\text { Mulder se retrouve dans } \\
\text { une boucle temporelle } \\
\text { dans laquelle il finit } \\
\text { chaque jour tué par un } \\
\text { braqueur de banque. }\end{array}$ & $\begin{array}{l}\text { Fictionnalité / distorsion } \\
\text { temporelle }\end{array}$ \\
\hline 6.19 & $\begin{array}{l}\text { The } \\
\text { Unnatural }\end{array}$ & $\begin{array}{l}\text { David } \\
\text { Duchovny ; } \\
\text { David } \\
\text { Duchovny }\end{array}$ & $\begin{array}{l}\text { Le frère du fondateur des } \\
\text { X-Files raconte l'histoire } \\
\text { d'un exceptionnel joueur } \\
\text { de baseball noir en } 1940 \\
\text { qui s'est révélé être un } \\
\text { extraterrestre. }\end{array}$ & $\begin{array}{l}\text { Fictionnalité / distorsion } \\
\text { temporelle ; } \\
\text { Transtextualité / } \\
\text { architextualité } \\
\text { (historique, film sportif) }\end{array}$ \\
\hline 6.20 & $\begin{array}{l}\text { Three of a } \\
\text { Kind }\end{array}$ & $\begin{array}{ll}\text { Bryan } & \text { Spicer } \\
\text { Vince } & \text { Gilligan } \\
\text { et John } & \text { Shibam }\end{array}$ & $\begin{array}{l}\text { Les Lone Gunmen } \\
\text { trompent Scully à les } \\
\text { rejoindre à Las Vegas } \\
\text { pour déjouer un complot. }\end{array}$ & $\begin{array}{l}\text { Fictionnalité / doubles et } \\
\text { mondes parallèles }\end{array}$ \\
\hline 7.12 & X-Cops & $\begin{array}{l}\text { Michael } \\
\text { Watkins ; } \\
\text { Vince Gilligan }\end{array}$ & $\begin{array}{l}\text { La traque d'une créature } \\
\text { qui prend la forme des } \\
\text { peurs de la victime pour } \\
\text { les tuer toutes les pleines } \\
\text { lunes dans un quartier } \\
\text { pauvre de L.A. }\end{array}$ & $\begin{array}{l}\text { Transtextualité / } \\
\text { intertextualité (pastiche } \\
\begin{array}{ll}\text { de la série } & \text { Cops) } \\
\text { architextualité } & \text { (télé- } \\
\text { réalité) } & \end{array}\end{array}$ \\
\hline 7.19 & $\begin{array}{l}\text { Hollywood } \\
\text { A.D. }\end{array}$ & $\begin{array}{l}\text { David } \\
\text { Duchovny ; } \\
\text { David } \\
\text { Duchovny }\end{array}$ & $\begin{array}{l}\text { Un scénariste ami de } \\
\text { Skinner fait un film de } \\
\text { série B hollywoodien } \\
\text { inspiré d'Ed Wood sur le } \\
\text { cas du bol de Lazare avec } \\
\text { Mulder et Scully. }\end{array}$ & $\begin{array}{l}\text { Narrativité/ points de vue } \\
\text { multiples et narrateurs } \\
\text { non fiables; } \\
\text { Transtextualité / } \\
\text { intertextualité (pastiche } \\
\text { etration); } \\
\text { Fictionnalité / doubles et } \\
\text { mondes parallèles }\end{array}$ \\
\hline 8.6 & Redrum & $\begin{array}{l}\text { Peter } \text { Markle; } \\
\text { Steven } \text { Maeda } \\
\text { et Daniel Arkin }\end{array}$ & $\begin{array}{l}\text { Un procureur ami de } \\
\text { Doggett est accusé du } \\
\text { meurtre de sa femme, } \\
\text { mais chaque jour il recule } \\
\text { de } 24 \text { heures, jusqu'au } \\
\text { jour du meurtre. }\end{array}$ & $\begin{array}{l}\text { Fictionnalité / distorsion } \\
\text { temporelle }\end{array}$ \\
\hline 9.18 & $\begin{array}{l}\text { Sunshine } \\
\text { Days }\end{array}$ & $\begin{array}{l}\text { Vince Gilligan ; } \\
\text { Vince Gilligan }\end{array}$ & $\begin{array}{l}\text { Un homme solitaire aux } \\
\text { puissants pouvoirs } \\
\text { psychokinésiques recrée } \\
\text { la maison et la famille de } \\
\text { The Brady Bunch. }\end{array}$ & $\begin{array}{l}\text { Transtextualité / } \\
\text { intertextualité (citation); } \\
\text { Narrativité / personnage } \\
\text { omnipotent }\end{array}$ \\
\hline
\end{tabular}


Buffy the Vampire Slayer (Joss Whedon, The WB, 1997-2001, UPN, 2001-2003)

\begin{tabular}{|c|c|c|c|c|}
\hline $\begin{array}{l}\text { Saison/ } \\
\text { épisode }\end{array}$ & $\begin{array}{l}\text { Titre de } \\
\text { l'épisode }\end{array}$ & Créateurs & Résumé & Tropes \\
\hline 1.10 & Nightmares & $\begin{array}{l}\text { Bruce Seth } \\
\text { Green; Joss } \\
\text { Whedon et } \\
\text { David } \\
\text { Greenwalt }\end{array}$ & $\begin{array}{l}\text { Un garçon dans le coma } \\
\text { transforme la réalité en } \\
\text { projetant les cauchemars } \\
\text { et les peurs de tous les } \\
\text { habitants de la ville. }\end{array}$ & $\begin{array}{l}\text { Fictionnalité / rêves, } \\
\text { inconscient ; } \\
\text { Narrativité / } \\
\text { personnage } \\
\text { omnipotent }\end{array}$ \\
\hline 2.2 & $\begin{array}{l}\text { Some Assembly } \\
\text { Required }\end{array}$ & $\begin{array}{lr}\text { Bruce } & \text { Seth } \\
\text { Green; } & \text { Ty } \\
\text { King } & \end{array}$ & $\begin{array}{l}\text { Un adolescent faire revivre } \\
\text { son grand frère star du } \\
\text { football en assemblant ses } \\
\text { membres à l'image de } \\
\text { Frankenstein. }\end{array}$ & $\begin{array}{l}\text { Transtextualité / } \\
\text { intertextualité } \\
\text { (hommage, pastiche) }\end{array}$ \\
\hline 2.6 & Halloween & $\begin{array}{l}\text { Bruce Seth } \\
\text { Green; Carl } \\
\text { Ellsworth }\end{array}$ & $\begin{array}{l}\text { Tous les habitants de la } \\
\text { ville se transforment en } \\
\text { leurs r déguisements } \\
\text { d'Halloween ensorcelés. }\end{array}$ & $\begin{array}{l}\text { Fictionnalité / doubles } \\
\text { et mondes parallèles; } \\
\text { Narrativité / } \\
\text { personnage } \\
\text { omnipotent }\end{array}$ \\
\hline 3.1 & Anne & $\begin{array}{l}\text { Joss } \\
\text { Whedon ; } \\
\text { Joss Whedon }\end{array}$ & $\begin{array}{l}\text { Buffy vit seule à Los } \\
\text { Angeles comme serveuse. }\end{array}$ & $\begin{array}{l}\text { Fictionnalité / doubles } \\
\text { et mondes parallèles }\end{array}$ \\
\hline 3.6 & Band Candy & $\begin{array}{l}\text { Michael } \\
\text { Lange; Jane } \\
\text { Espenson }\end{array}$ & $\begin{array}{l}\text { Des friandises ensorcelées } \\
\text { transforment tous les } \\
\text { adultes de Sunnydale en } \\
\text { adolescents. }\end{array}$ & $\begin{array}{l}\text { Fictionnalité / doubles } \\
\text { et mondes parallèles; } \\
\text { Narrativité / } \\
\text { personnage } \\
\text { omnipotent }\end{array}$ \\
\hline 3.9 & The Wish & $\begin{array}{l}\text { David } \\
\text { Greenwalt ; } \\
\text { Marti Noxon }\end{array}$ & $\begin{array}{l}\text { Cordelia fait le vœu que } \\
\text { Buffy ne soit jamais venue } \\
\text { à Sunnydale et altère ainsi } \\
\text { la réalité. }\end{array}$ & $\begin{array}{l}\text { Fictionnalité / doubles } \\
\text { et mondes parallèles; } \\
\text { Narrativité / } \\
\text { personnage } \\
\text { omnipotent }\end{array}$ \\
\hline 3.16 & Doppelgangland & $\begin{array}{l}\text { Joss } \\
\text { Whedon ; } \\
\text { Joss Whedon }\end{array}$ & $\begin{array}{l}\text { Willow-vampire, issue du } \\
\text { monde parallèle créé dans } \\
\text { l'épisode } \\
\text { «Doppengangland», est de } \\
\text { retour. }\end{array}$ & $\begin{array}{l}\text { Fictionnalité / doubles } \\
\text { et mondes parallèles }\end{array}$ \\
\hline 4.9 & Something Blue & $\begin{array}{l}\text { Nick Mark; } \\
\text { Tracey } \\
\text { Forbes }\end{array}$ & $\begin{array}{l}\text { Voulant apaiser sa peine } \\
\text { d'amour, Willow jette un } \\
\text { sort qui a pour } \\
\text { conséquence que tout ce } \\
\text { qu'elle dit devient réalité. }\end{array}$ & $\begin{array}{l}\text { Fictionnalité / doubles } \\
\text { et mondes parallèles; } \\
\text { Narrativité / } \\
\text { personnage } \\
\text { omnipotent }\end{array}$ \\
\hline
\end{tabular}




\begin{tabular}{|c|c|c|c|c|}
\hline 4.10 & Hush & $\begin{array}{l}\text { Joss } \\
\text { Whedon ; } \\
\text { Joss Whedon }\end{array}$ & $\begin{array}{l}\text { Des démons volent la } \\
\text { capacité de parler de tout } \\
\text { Sunnydale. }\end{array}$ & $\begin{array}{l}\text { Transtextualité / } \\
\text { architextualité (conte } \\
\text { de fées, cinéma muet) }\end{array}$ \\
\hline 4.17 & Superstar & $\begin{array}{l}\text { David } \\
\text { Grossman ; } \\
\text { Jane } \\
\text { Espenson }\end{array}$ & $\begin{array}{l}\text { Jonathan altère la réalité } \\
\text { avec un sortilège pour être } \\
\text { une super-vedette capable } \\
\text { de tout faire, notamment à } \\
\text { la place de Buffy. }\end{array}$ & $\begin{array}{l}\text { Fictionnalité / doubles } \\
\text { et mondes parallèles ; } \\
\text { Narrativité / } \\
\text { personnage } \\
\text { omnipotent }\end{array}$ \\
\hline 4.22 & Restless & $\begin{array}{l}\text { Joss } \\
\text { Whedon; } \\
\text { Joss Whedon }\end{array}$ & $\begin{array}{l}\text { La «Scooby gang" se } \\
\text { retrouve coincé dans un } \\
\text { cauchemar, traqué par la } \\
\text { première slayer. }\end{array}$ & $\begin{array}{l}\text { Fictionnalité / rêves, } \\
\text { inconscient }\end{array}$ \\
\hline 5.1 & Buffy vs Dracula & $\begin{array}{l}\text { David } \\
\text { Solomon ; } \\
\text { Marti Noxon }\end{array}$ & $\begin{array}{l}\text { Dracula arrive à Sunnydale } \\
\text { et ensorcelle Buffy. }\end{array}$ & $\begin{array}{l}\text { Transtextualité / } \\
\text { intertextualité } \\
\text { (hommage, pastiche) }\end{array}$ \\
\hline 5.16 & The Body & $\begin{array}{l}\text { Joss } \\
\text { Whedon ; } \\
\text { Joss Whedon }\end{array}$ & $\begin{array}{l}\text { Buffy trouve le corps de sa } \\
\text { mère morte d'un } \\
\text { anévrisme et doit gérer la } \\
\text { situation. }\end{array}$ & $\begin{array}{l}\text { Fictionnalité / } \\
\text { distorsion temporelle ; } \\
\text { Transtextualité / } \\
\text { architextualité } \\
\text { (hyperréalisme) }\end{array}$ \\
\hline 6.5 & Life Serial & $\begin{array}{l}\text { Nick Marck; } \\
\text { David Fury et } \\
\text { Jane } \\
\text { Espenson }\end{array}$ & $\begin{array}{l}\text { Le trio teste Buffy, } \\
\text { notamment en la plaçant } \\
\text { dans une } \quad \text { boucle } \\
\text { temporelle infinie. }\end{array}$ & $\begin{array}{l}\text { Fictionnalité / } \\
\text { distorsion temporelle ; } \\
\text { Narrativité / } \\
\text { personnage } \\
\text { omnipotent }\end{array}$ \\
\hline 6.7 & $\begin{array}{l}\text { Once More With } \\
\text { Feeling }\end{array}$ & $\begin{array}{l}\text { Joss } \\
\text { Whedon ; } \\
\text { Joss Whedon }\end{array}$ & $\begin{array}{l}\text { Un démon transforme } \\
\text { Sunnydale en comédie } \\
\text { musicale. }\end{array}$ & $\begin{array}{l}\text { Transtextualité / } \\
\text { architextualité } \\
\text { (comédie musicale); } \\
\text { Narrativité / } \\
\text { personnage } \\
\text { omnipotent ; } \\
\text { Fictionnalité / mondes } \\
\text { parallèles }\end{array}$ \\
\hline 6.8 & Tabula Rasa & $\begin{array}{l}\text { David } \\
\text { Grossman ; R. } \\
\text { R. Kirshner }\end{array}$ & $\begin{array}{l}\text { Un sortilège de Willow } \\
\text { tourne mal et provoque } \\
\text { l'amnésie totale de tous. }\end{array}$ & $\begin{array}{l}\text { Fictionnalité / doubles } \\
\text { et mondes parallèles; } \\
\text { Narrativité / } \\
\text { personnage } \\
\text { omnipotent }\end{array}$ \\
\hline
\end{tabular}




\begin{tabular}{|c|c|c|c|c|}
\hline 6.17 & Normal Again & $\begin{array}{l}\text { Rick } \\
\text { Rosenthal ; } \\
\text { Diego } \\
\text { Gutierrez }\end{array}$ & $\begin{array}{l}\text { Contaminé par un démon, } \\
\text { Buffy est convaincue que la } \\
\text { réalité n'est qu'une } \\
\text { hallucination lorsqu'elle se } \\
\text { réveille dans un hôpital } \\
\text { psychiatrique. }\end{array}$ & $\begin{array}{l}\text { Fictionnalité / doubles } \\
\text { et mondes parallèles; } \\
\text { rêves, inconscient } \\
\text { (folie) }\end{array}$ \\
\hline 7.7 & \begin{tabular}{l}
\multicolumn{2}{l}{ Conversation } \\
With Dead \\
People
\end{tabular} & $\begin{array}{l}\text { Nick Marck; } \\
\text { Jane } \\
\text { Espenson et } \\
\text { Drew } \\
\text { Goddard }\end{array}$ & $\begin{array}{l}\text { Rencontres en temps réel } \\
\text { entre chaque membre de la } \\
\text { «Scooby Gang » isolé et un } \\
\text { personnage mort dans la } \\
\text { série. }\end{array}$ & $\begin{array}{l}\text { Narrativité / points de } \\
\text { vue multiples et } \\
\text { narrateurs non } \\
\text { fiables; Fictionnalité / } \\
\text { distorsion temporelle }\end{array}$ \\
\hline 7.16 & Storyteller & $\begin{array}{l}\text { Marita } \\
\text { Grabiak } \\
\text { Jane } \\
\text { Espenson }\end{array}$ & $\begin{array}{l}\text { Andrew se réfugie dans les } \\
\text { toilettes et se filme en } \\
\text { train de raconter sa vision } \\
\text { fantasmée et décalée de } \\
\text { l'histoire de la saison. }\end{array}$ & $\begin{array}{l}\text { Narrativité / points de } \\
\text { vue multiples et } \\
\text { narrateurs non } \\
\text { fiables; Fictionnalité } \\
\text { / } r \text { distorsion } \\
\text { temporelle }\end{array}$ \\
\hline
\end{tabular}

\section{NOTES}

1. Danielle Aubry, Du roman-feuilleton à la série télévisuelle. Pour une rhétorique du genre et de la sérialité, Berne, Peter Lang, 2006, p. 135.

2. Stéphane Benassi, Séries et feuilletons T.V.: Pour une typologie des fictions télévisuelles, Liège, Éditions du CÉFAL, 2000, p. 9.

3. Benassi, p. 9.

4. Dans son article publié dans ce dossier ( « La forme semi-feuilletonnante formulaire : l'exemple d'Ally McBeal ", TV/Series, no 15, 2019), Claire Cornillon parle quant à elle de "semifeuilletonnant formulaire " pour désigner ces séries hybrides qui commencent à apparaittre dans les années 1990.

5. Jean-Pierre Esquenazi, Les Séries télévisées : l'avenir du cinéma ?, $2^{\mathrm{e}}$ édition, Paris, Armand Colin, 2014, p. 94.

6. Stéphane Benassi, « Sérialité(s) et esthétique de la fiction télévisuelle », Belphégor, Volume 14, n ${ }^{\circ} 1,2016$, \$2, https://journals.openedition.org/belphegor/770?lang=en.

7. Guillaume Soulez, «La double répétition : structure et matrice des séries télévisées », Mise au point, $\mathrm{n}^{\circ} 2,2011, \S 1$, https://journals.openedition.org/map/979\#ftn3.

8. Soulez, §8.

9. Noël Nel, «Téléfilm, feuilleton, série, saga, sitcom, soap-opera, telenovela : quels sont les éléments clés de la sérialité ? ", CinémAction, n57, octobre 1990, p. 64.

10. Jason Mittell, Complex TV: The Poetics of Contemporary Televion Storytelling, New York, NYU Press, 2015, p. 18.

11. À ce sujet, voir surtout Robert Thompson, Television's Second Golden Age: From Hill Street Blues to ER, Syracuse (NY), Syracuse University Press, 1997.

12. Esquenazi, p. 120.

13. Voir à ce sujet, Tzvetan Todorov, «Typologie du roman policier », in Poétique de la prose, Paris, Seuil, 1971, p. 60.

14. Alain Carrazé et Romain Nigita, Les Nouveaux feuilletonistes: Ils ont révolutionné les séries TV, Paris, Fantask, 2016, p. 329-330. 
15. Ce processus d'hybridation est présent dans de nombreuses séries des années 1990, et les éléments appartenant à l'une ou l'autre des deux formes ne sont pas toujours aussi aisément identifiables, ce que nous révèle Claire Cornillon (op. cit.) dans son étude d'Ally McBeal en tant que semi-feuilleton formulaire. C'est d'ailleurs le propre de l'hybridité que d'offrir des résultats polymorphes et plus ou moins identifiable, selon les cas.

16. Mittell, p. 19-20.

17. Ce type d'épisode ne fait pas l'unanimité auprès des spectateurs, certains y voyant simplement du remplissage. Florent Favard parle plus positivement de "délayage narratif » ( Écrire une série TV: la promesse d'un dénouement, Tours, Presses universitaires François Rabelais, 2019, p. 219), suggérant qu'un récit a parfois besoin de respirer, d'espace pour se déployer. Cette notion est particulièrement intéressante dans une perspective structurelle et narrative, mais j'avance que les épisodes autonomes dont je parle ici offrent une pertinence dans les séries qui n'est justement pas (que) narrative.

18. Juliette Harrisson, "The X-Files: A Binge Guide for Beginners », Den of Geek!, 28 décembre 2017, http://www.denofgeek.com/us/tv/the-x-files/244152/the-x-files-a-binge-guide-forbeginners.

19. Mittell, p. 46.

20. Aubry, p. 128.

21. Ces trois notions ont été largement travaillées par Gérard Genette (Fiction et diction [Paris, Seuil, 1991] pour la fictionnalité, Discours du récit [Paris, Seuil, 2007] pour la narrativité, Palimpsestes: la littérature au second degré [Paris, Seuil, 1982] pour la transtextualité) et par bien d'autres depuis. Sans entrer dans les nombreux débats théoriques que la notion de "fiction » a pu générer (voir à ce sujet René Audet et Alexandre Gefen, (dir.), Frontières de la fiction, Québec et Bordeaux, Nota Bene et Presses Universitaires de Bordeaux, 2001), la « fictionnalité » dans le sens utilisé ici évoque la cohérence, les frontières et la porosité du monde fictionnel mis en scène, la relation qu'il établit avec le réel et avec d'autres mondes fictionnels internes (mondes parallèles, rêve, fantasme, etc.). La narrativité se définit de manière assez classique par la façon dont une histoire est racontée, que ce soit sur le plan structurel, de point de vue (voix narrative), de temps, de vitesse, etc. Enfin, la transtextualité selon Genette est « tout ce qui met un texte en relation, manifeste ou secrète, avec un autre texte" (Genette, 1982, p. 7). Il existe plusieurs types de relation en fonction du niveau de proximité, de présence et de transparence : l'intertextualité, la paratextualité, la métatextualité, l'hypertextualité et l'architextualité.

Certains tropes pourraient évidemment être classés dans plus d'une catégorie. Par exemple, la mise en scène de mondes parallèles fait également partie d'une réflexion sur la narrativité, en particulier si ces mondes parallèles sont étanches et servent de stratégie narrative (voir Mr. Nobody, Jaco Van Dormael, 2009), mais dans les séries de l'imaginaire, ces mondes parallèles fictifs sont le plus souvent poreux et visent une réflexion et un jeu sur la nature même de la réalité (voir Buffy, « The Wish », S03E09).

22. Jorge Luis Borges, « Le jardin aux sentiers qui bifurquent », in Fictions, Paris, Gallimard, (1941) 1983, p. 91-104.

23. «"Groundhog Day" Loop », TV Tropes, consultée le 20 mai 2018, http://tvtropes.org/pmwiki/ pmwiki.php/Main/GroundhogDayLoop.

24. À propos de la théorie des mondes possibles et ra riche utilisation en fiction, notamment littéraire, mais aussi télésérielle, lire le collectif dirigé par Françoise Lavocat, La théorie littéraire des mondes possibles (Paris, CNRS Éditions, 2010, 324 p.), et en particulier le chapitre de MarieLaure Ryan, « Cosmologie du récit des mondes possibles aux univers parallèles » (p. 53-83).

25. Emily VanDerWerff, " 10 Must-See Episodes of The X-Files», AV/TV Club, 20 juillet 2012, https://tv.avclub.com/10-must-see-episodes-of-the-X-files-1798232355.

26. Carrazé, p. 350.

27. Mittell, p. 46-47. 
28. Du côté, des critiques, les épisodes autonomes se retrouvent régulièrement au sommet des palmarès des meilleurs épisodes. Par exemple, le magazine Empire et ses critiques Nick de Semlyen et James White placent dans leur top 20 des meilleurs épisodes de The X-Files (https:// www.empireonline.com/movies/features/greatest-X-files-episodes/) sept épisodes autonomes: "X-Cops ", « Musings of A Cigarette Smoking-Man», «Bad Blood», «Triangle ", "Drive ", «Ice », « The Post-Modern Prometheus ». De son côté, Jennifer Armstrong du Rolling Stone place dans son top 10 de Buffy (https://www.rollingstone.com/tv/tv-lists/10-best-buffy-the-vampireslayer-episodes-of-all-time-113273/) quatre épisodes autonomes : « The Wish », « Hush », " Once More With Feeling » et « The Body ». Les palmarès de ce type sont légion et présentent en général le même ratio d'épisodes autonomes. Ils offrent souvent l'occasion aux fans de voter pour leur épisode favori. Pour Buffy, il s'agit presque toujours de "Once More With Feeling », «Hush » ou "The Body ». Pour The X-Files, "Ice », "Bad Blood ", " The Post-Modern Prometheus », "Jose Chung's "From Outer Space" » et « Triangle » sont toujours nommés parmi les 10 ou 15 préférés.

29. Certains épisodes autonomes ont pu recevoir de nombreuses nominations et prix, notamment des Emmy (l'épisode « The Post-Modern Prometheus » de The X-Files; les épisodes de Buffy " Once More With Feeling » et « Hush »), mais aussi de nombreux prix de l'industrie : les associations des American Cinema Editors et des Motion Picture Sound Editors, la Cinema Audio Society, la Directors Guild of America et la Online Film \& Television Association. Mentionnons aussi des prix spécifiques aux genres de l'imaginaire comme le Bram Stoker Awards; les Hugo Awards; la Science Fiction and Fantasy Writers of America et les SFX Awards.

30. Les entrevues avec les créateurs, notamment celles réalisées par Alain Carrazé (publié dans son ouvrage Les nouveaux feuilletonistes en 2016) en témoignent.

31. Monica Michlin, « More, More, More: Contemporary American TV Series and the Attractions and Challenges of Serialization as Ongoing Narrative ", Mise au point, no 3, 2011, §1, https:// journals.openedition.org/map/927.

32. Esquenazi, p. 110.

33. Gérard Genette, Figures V, Paris, Seuil, 2002, p. 85-86.

34. Favard, p. 141.

\section{RÉSUMÉS}

Les genres télésériels évoluent depuis l'émergence du médium, ils se raffinent, se complexifient, s'hybrident, répondant aux exigences et aux compétences spectatorielles grandissantes de son public. Les genres permettent ainsi l'émergence de formules, que l'on adopte pour mieux se les approprier, et à partir desquelles les créateurs peuvent construire la matrice qui donnera à leur œuvre sa structure unique. Entre les séries canoniques des débuts et les grands feuilletons d'aujourd'hui, les années 1990 ont donné naissance à plusieurs séries hybrides, notamment dans le sous-genre du fantastique (par exemple, The X-Files et Buffy The Vampire Slayer), qui combinent des épisodes sériels et feuilletonnants. Au cœur de ces séries se trouvent des épisodes autonomes, souvent les plus appréciés, qui s'écartent de la matrice et proposent des innovations ou des jeux fictionnels, narratifs ou transtextuels.

TV serial genres have evolved since the emergence of the medium, they are becoming more refined, more complex, more hybrid, responding to the growing demands and spectatorial skills of its audience. The genres thus allow the emergence of formulas, which are appropriated by 
creators, and from which they can build the matrix that will give their work its unique structure. Between the early canonical series and today's great serials, the 1990s gave rise to several hybrid series, notably in the fantasy subgenre (for example, The X-Files and Buffy The Vampire Slayer), which combine serial episodes and larger seasonal story arc. At the heart of these series are stand-alone episodes, often the most appreciated, that move away from the matrix and propose fictional, narrative or transtextual innovations and games.

\section{INDEX}

Mots-clés : épisodes autonomes, matrice, formule, feuilleton, sérialité, narrativité, fictionnalité, transtextualité, genre, séries fantastiques et de science-fiction, Buffy contre les vampires, XFiles : Aux frontières du réel

Keywords : stand-alone episodes, matrix, formula, TV serials, seriality, narrativity, fictionnality, transtextuality, genre, SF and Fantasy TV series, Buffy the Vampire Slayer, X-Files (The)

\section{AUTEUR}

\section{ELAINE DESPRÉS}

Elaine Després est chercheure indépendante et coordonnatrice de Figura, Centre de recherche sur le texte et l'imaginaire, situé à l'Université du Québec à Montréal, où elle a obtenu son doctorat (2012). Elle a publié Pourquoi les savants fous veulent-ils détruire le monde ?Évolution d'une figure littéraire (Le Quartanier, 2016) et codirigée des ouvrages collectifs sur le posthumain (PUR, 2014 ; Cahiers du CEIMA, 2013) et les séries télévisées contemporaines (Revue Otrante, 2017 ; XYZ, 2017). Ses recherches portent sur les représentations imaginaires de la science dans une perspective sociocritique.

Elaine Després is an independent researcher and the coordinator of the Figura research center at the Université du Québec à Montréal, where she defended her thesis in 2012. She has published a book about the mad scientist trope in literature entitled Pourquoi les savants fous veulent-ils détruire le monde ? Évolution d'une figure littéraire (Le Quartanier, 2016) and co-edited collective works about the posthuman (PUR, 2014 ; Cahiers du CEIMA, 2013) and contemporary TV series (Revue Otrante, 2017; XYZ, 2017). Her research mainly concerns the representations of science in fiction, informed by sociological criticism. 